\title{
Endovascular and Percutaneous Management of the Pseudoaneurysms
}

\author{
Sahu Chandradev, Singhal Ateesh \\ Department of Radiodiagnosis, Pt. Jawahar Lal Nehru Memorial Medical College and Associated Dr. Bhim Rao \\ Ambedkar Memorial Hospital, Raipur, India \\ Email: cdsahu78@gmail.com
}

Received 12 June 2014; revised 17 July 2014; accepted 1 August 2014

Copyright (C) 2014 by authors and Scientific Research Publishing Inc.

This work is licensed under the Creative Commons Attribution International License (CC BY). http://creativecommons.org/licenses/by/4.0/

(c) (i) Open Access

\begin{abstract}
Introduction: Pseudoaneurysm is a dilatation of an artery with actual disruption of one or more layers of its wall, rather than just expansion of wall layers. Pseudoaneurysms are common vascular abnormalities. Computed Tomography (CT), Magnetic Resonance Imaging (MRI), sonography, and angiography all are valuable modalities in the imaging workup of pseudoaneurysms. Conventional angiography remains the gold standard for diagnosis. Therapeutic options have evolved in recent years from the traditional surgical option toward a less invasive approach which includes procedures such as Ultrasound (US)-guided compression, US-guided percutaneous thrombin injection, and endovascular management (embolization, stent-graft placement and endovascular coiling). Objective: In this article, we discuss pseudoaneurysms of various arteries, and available percutaneous and endovascular treatment options. Materials and Methods: Sixteen patients diagnosed with pseudoaneurysm involving visceral and peripheral arteries were included in this study. The initial diagnosis was made with CDUS, CECT, and CTA followed by catheter angiogram for therapeutic procedures. Results: All the 16 patients with pseudoaneurysms were successfully treated with endovascular and percutaneous embolization to achieve stasis. Conclusion: CT, MRI, sonography, and angiography may all be valuable in the imaging workup of pseudoaneurysms. The use of minimally invasive treatment has led to a marked decrease in the morbidity and mortality rates for pseudoaneurysms.
\end{abstract}

\section{Keywords}

Pseudoaneurysm, Endovascular, Percutaneous, Embolization, Computed Tomographic Angiography (CTA)

\section{Introduction}

Pseudoaneurysms are common vascular abnormalities that represent a disruption in the arterial wall continuity.

How to cite this paper: Chandradev, S. and Ateesh, S. (2014) Endovascular and Percutaneous Management of the Pseudoaneurysms. Open Journal of Radiology, 4, 241-254. http://dx.doi.org/10.4236/ojrad.2014.43032 
The associated complications have high morbidity and mortality rates. Conventional angiography remains the standard of reference for diagnosis which is invasive procedure, but non-invasive diagnostic modalities e.g., magnetic resonance angiography (MRA), computed tomographic angiography (CTA) or/and ultrasonography [US] should be included in the initial work-up if possible. The less invasive therapeutic options have evolved in recent years which include radiologic procedures such as Ultrasound (US)-guided compression, US-guided percutaneous thrombin injection, and endovascular management (embolization, stent-graft placement, entrapment and coiling). The recent treatment options have led to a significant decrease in the morbidity and mortality.

Pseudoaneurysms can develop in all the arteries of the human body. Common etiological factors are major trauma, pancreatitis, iatrogenic, syphilis, Marfan syndrome, or infection. Atherosclerotic aneurysms are often seen in large arteries and in patients of advanced age, but pseudoaneurysms due to penetrating or blunt trauma are seen in patients of any age and location.

\section{Materials and Methods}

\subsection{Patients}

16 patients presented to our department with different clinical complaints are included.

\subsection{Material Used}

\subsubsection{Imaging Systems}

Ultrasound-Toshiba Aplio MX.

CT-Siemens Somatom Definition AS+ 128 slice CT system.

MR-3T Siemens Magnetom Skyra.

0.2T Siemens Magnetom Concerto.

DSA-GE Innova 4100 Cath-Angio System $41 \mathrm{~cm}$ by $41 \mathrm{~cm}$ Flat panel detector (Milwaukee, USA).

\subsubsection{Interventional and Therapeutic Materials}

5F C1 Cobra catheter (assembled in Mexico Cordis Corporation Miami lakes Florida USA), Axium Microcoils, Eschelon 10, $45^{\circ}$, Xpedion eV3 (Microtherapeutics, Inc d/b/a ev3 Neurovascular Irvine, California), Axium detachable coil system with Covidien's unique Instant Detacher (USA) Plymouth, MN USA. Baxter AG human thrombin (Vienna, Austria), 0.35 guidewire (Terumo Coprporation Tokyo, Japan), Endocryl (n-butyl cyanoacrylate) (Nalagarh Solan, Himachal Pradesh), Hilal and Nester Platinum Embolization Coils (Cook Medical Inc., Bloomington, IL, USA), Lipidol Ultra-Fluide (Aulna-sous-Bois France).

\subsection{Embolization Technique}

The right femoral artery was punctured. With the help of $5 \mathrm{~F}$ angiographic catheter and guide wire selective catheterization of the feeding artery was done. Initial were taken and carefully assessed for the location, size, neck size and rupture if present of the pseudoaneurysms and then the appropriate mode of the treatment were opted to achieve complete occlusion.

\subsubsection{Endovascular Technique}

Through the same route with super-selective catherization, embolizing agents and/or microcoils were inserted carefully under DSA control to exclude the pseudo aneurysm from circulation.

\subsubsection{Percutaneous Technique}

Under USG guidance direct percutaneous puncture was performed with 21 Gauge needle. The location was confirmed with direct introduction of the contrast under fluoroscopic guidance followed by introduction of human thrombin/glue in the psuedoaneurysmal sac.

\section{Observations}

A total of 16 patients were included in this study with M:F ratio of 7:1. The initial diagnosis was confirmed with the help of Colour Doppler Ultrasonography (CDUS), Contrast Enhanced CT (CECT) scan, CTA followed by 
catheter angiogram for therapeutic procedures. The table (Table 1) shows the individual case details in the form of the cause of pseudoaneurysm, arterial site of involvement, embolizing agent used and final outcome.

\section{Discussion}

Pseudoaneurysm is one in which the wall of the artery is perforated with focal dilatation.

\subsection{Clinical Features}

Pseudoaneurysms may be asymptomatic and detected incidentally during radiologic procedures for other etiologies. Most of the patients with visceral artery aneurysms (VAA) are often asymptomatic (72\%) [1]. Symptomatic pseudoaneurysms manifest with local or systemic signs and symptoms. Local effects of a pseudoaneurysm are secondary to mass effect on adjacent structures. They may manifest as a palpable thrill, audible bruit, or pulsatile mass. Ischemia of the surrounding tissues due to vascular compromise may lead to necrosis of the organ and adjacent tissue. Thromboembolism is also a potential complication. In addition, rupture of pseudoaneurysm leads to lethal complications such as hypo-volumic shock, hematemesis, malena or SAH.

\subsection{Diagnosis}

There are various modalities available to confirm the diagnosis of pseudoaneurysm which include non-invasive and invasive modalities. Non-invasive methods includes CDUS, CTA and MRA, and Non-invasive methods includes catheter angiogram. Non-invasive methods is the preferred modality to make the diagnosis. However in some special cases the non-invasive modalities do not reveal pseudoaneurysm, then catheter angiogram should be done (Figure 1).

\section{Table 1. Individual case detail.}

\begin{tabular}{|c|c|c|c|c|c|}
\hline S. No & Cause & Location & $\begin{array}{c}\text { Location wise } \\
\text { number of cases (n) }\end{array}$ & Embolizing agents & Outcome/comments \\
\hline 1 & Iatrogenic & Right hepatic artery & \multirow{4}{*}{4} & Glue & None \\
\hline 2 & Iatrogenic & Right hepatic artery & & Glue & None \\
\hline 3 & Pancreatitis & Right hepatic artery & & Coil + glue & None \\
\hline 4 & Pancreatitis & Common Hepatic artery & & Glue & None \\
\hline 5 & Trauma & Left posterior cerebral artery & \multirow{3}{*}{3} & Coils + onyx & $\begin{array}{l}\text { Recurrence followed by } \\
\text { parent artery occlusion }\end{array}$ \\
\hline 6 & Trauma & Right internal carotid artery (ICA) & & Coils & $\begin{array}{l}\text { Left hemiparesis followed } \\
\text { by complete recovery }\end{array}$ \\
\hline 7 & Iatrogenic & Right ICA & & Coils & None \\
\hline 8 & Trauma & Left brachial artery & \multirow[b]{2}{*}{2} & Human thrombin & None \\
\hline 9 & Trauma & Right ulnar artery & & Glue & None \\
\hline 10 & Iatrogenic & Left renal artery & \multirow[b]{2}{*}{2} & Glue & None \\
\hline 11 & Iatrogenic & Left renal artery & & Glue & None \\
\hline 12 & Pancreatitis & Gastroduodenal artery & \multirow[b]{2}{*}{2} & Human thrombin + coils & None \\
\hline 13 & Pancreatitis & Splenic artery & & Coil + glue & None \\
\hline 14 & Trauma & Transverse facial artery & 1 & Glue & None \\
\hline 15 & Iatrogenic & Right uterine artery & 1 & Glue & None \\
\hline 16 & Iatrogenic & Right ilio-colic artery & 1 & Glue & None \\
\hline
\end{tabular}




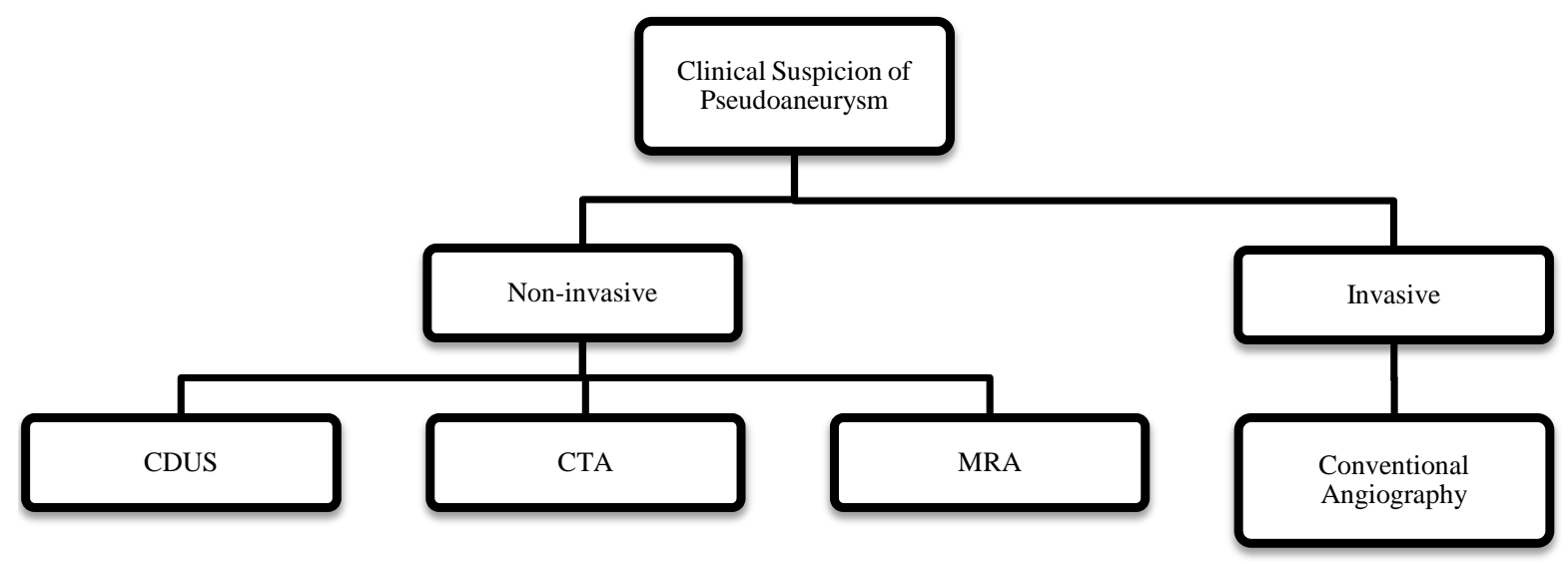

Figure 1. Modalities available for the diagnosis of the pseudoaneurysms.

\subsection{Treatment}

Although surgery was the gold-standard treatment in the past, several less invasive treatment options are more popular today [1] [2].

\subsubsection{Ultrasound-Guided Compression}

Ultrasound-guided probe compression of the neck of the pseudoaneurysm is a novel non-invasive method of treatment with narrow neck. The ultrasound probe is pushed firmly against the neck of the pseudoaneurysm usually for about 20 minutes. The blood within the pseudoaneurysm clots during this time interval. This procedure needs to be repeated multiple times. It is less successful in wide neck pseudoaneurysms, obese patients and patient on anticoagulant. It is the least invasive method to stop arterial bleeding.

\subsubsection{Ultrasound-Guided Thrombin Injection}

Under ultrasound guidance, thrombin can be injected directly into a pseudoaneurysm which leads to rapid clot formation. Thrombin (factor IIa in the coagulation cascade) is a clotting factor that converts fibrinogen into fibrin, which then polymerizes to form a blood clot. The technique is relatively easy to perform and is minimally invasive.

\subsubsection{Endoluminal Management}

Endoluminal management serves to exclude a pseudoaneurysm from the circulation. Treatment option depends on the size of the pseudoaneurysmal neck and the expendability of the donor artery (Figure 2). Exclusion methods fall into two broad categories: embolization and stent placement.

A pseudoaneurysm that arises from an expendable donor artery and does not have a collateral supply such as a visceral branch (e.g., a segmental or intra-lobar renal arterial branch) is treated with embolization of the afferent artery. There are visceral arteries that have a well-established collateral supply, such as gastroduodenal, hepatic, and splenic arteries and other upper gastrointestinal arteries. Some pelvic and distal extremity arteries also have good collateral supply-such as the internal iliac arteries and the profunda femoris arteries. When embolizing arteries with numerous collateral vessels, one must embolize both proximal and distal to the pseudoaneurysm to completely exclude it from the circulation by preventing backflow from the collateral circulation.

Pseudoaneurysm arising from an in-expendable artery must be excluded from the circulation while preserving the donor artery. The width of the pseudoaneurysmal neck is the key determining factor in this method. If the vital donor artery has to be embolized in certain emergent situations then distal flow must be restored by means of a surgical bypass procedure to prevent end organ damage.

If the neck is narrow, the sac may be embolized with catheter-directed delivery of coils itself. Coils can be non-detachable and detachable. With the availability of newer platinum based coils which are softer, and have a more complex (helical/3D) shape; conforming to the shape of sac allowing use of fewer coils for embolization. However, because platinum coils are softer, initial placement of stainless steel coils may be required to act as scaffolding. Use of detachable coils facilitates more accurate deployment and the possibility of readjustment 


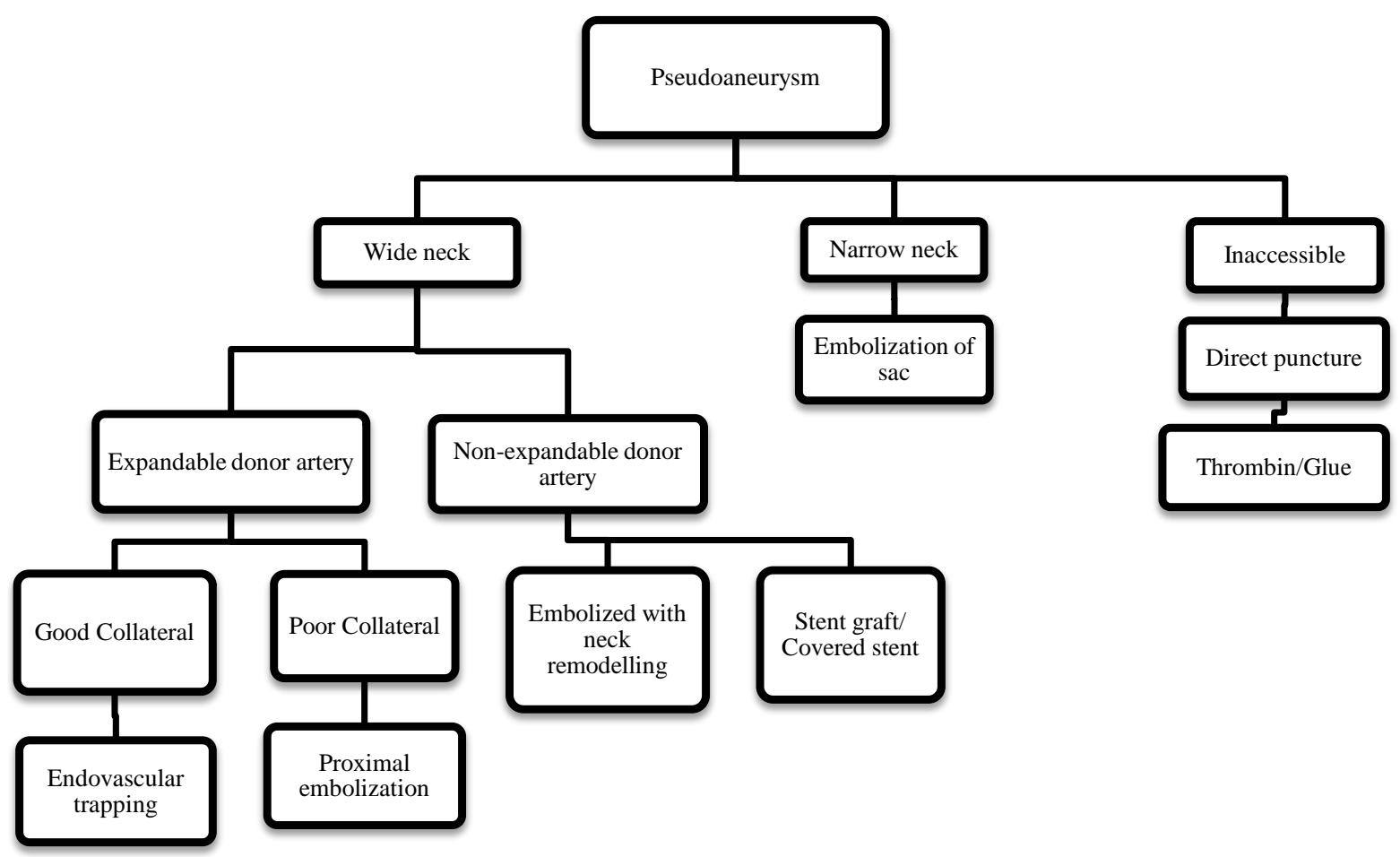

Figure 2. Algorithmic illustration for the treatment of the pseudoaneurysms.

before its final deployment. Potential disadvantage of coils in the pseudoaneurysmal sac is recanalization which is overcome by the use of soft helical coils. Other agents such as thrombin or N-butyl 2-cyanoacrylate (glue) may be used, either alone or in addition to coils.

If the neck is wide, the pseudoaneurysm can still be embolized with catheter-directed delivery of embolizing materials. However, remodelling is required to prevent outflow of these materials into distal donor artery and to ensure adequate embolization of the pseudoaneurysmal sac. This remodelling may be performed with stent cage, stent assisted coiling or flow diverter. If distal arterial embolization is a concern, detachable balloons may be used as the embolic agent. Another option, if the pseudoaneurysmal neck is wide, is stent-graft (covered stent) placement across the neck to exclude the pseudoaneurysm. This method is relatively contraindicated in mycotic pseudoaneurysms due to potential stent-graft infection. Visceral pseudoaneurysms, which are usually smaller and located off small and tortuous donor arteries, pose a particular challenge for stent-graft placement.

Endovascular techniques have a lower complication rate than does surgical management. Complications associated with endovascular techniques include intra-procedural rupture of the pseudoaneurysm, recanalization of the embolized vessel and reconstitution of arterial flow to the pseudoaneurysm (delayed failure of embolization).

Various embolic agents are available such as Ethanol, gel foam particles, Poly Vinyl Alcohol (PVA), microcoil, detachable balloons and N-butyl-2-cyanoacrylate [3]. Intra-arterial ethanol injection reduces the vascularity of vascular lesions to facilitate their surgical resection; selective arterial injection induces luminal thrombosis with endothelial loss with varying degrees of medial necrosis, leading to its occlusion. Temporary embolizing agents such as PVA and Gel-foam has several limitations which includes: 1) reflux of embolic material into the normal arteries, particularly if a small distal vessel has not been super-selectively cannulated, 2) a larger vessel may be difficult to occlude, 3) recanalization of the vessel due to resorption of the embolizing agents. In moderate-sized vessels, steel coils or detachable balloons may also be used. Cyanoacrylic glue is a permanent efficiently occluding agent that has been successfully used for more than 20 years in the endoluminal treatment of vascular cerebral malformation [3]. According to Soyer et al. [3], the use of n butyl-2-cyanoacrylate is less expensive than the microcoil.

\subsubsection{Surgical Ligation (with or without Distal Bypass)}

Open surgery may also be performed to remove pseudoaneurysms or prevent them from expanding. However, it 
is more invasive and, there is more postoperative pain and risk for wound infection. One of the less invasive options may be preferred in a patient with many co morbidities, which is at high risk for surgery.

Hepatic artery pseudoaneurysms (HAPA) are rare. However, rupture is common and occurs in $76 \%$ of patients [4]. The right hepatic artery is the most common artery involved. Hepatic, biliary or pancreatic procedures (e.g. liver biopsy, cholecystectomy, hepatectomy, liver transplantation and biliary trans-hepatic drainage) can cause HAPA. Although pseudoaneurysm may present with epigastric or subcostal pain, the most common presentation is bleeding that may present with rupture several months to years after undergoing any of these procedures. Duodenoscopy is useful for detecting bleeding from the papilla in many patients. Doppler ultrasonography can detect HAPA, but the most useful diagnostic procedure is catheter angiogram. There are different options available for the treatment however the factors that affect treatment choice are institution's preference, general condition of the patient, morphological characteristics and the anatomic location of the lesions. HAPA with different causes were treated by endovascular embolization (Figure 3). Surgical options are reserved for those who fail embolization [5].

One of our patients presented with hematemesis, malena and recurrent abdominal pain. His history revealed surgical drainage of large liver abscess 2 months ago. After stabilization, emergency esophagogastroduodenoscopy was performed which blood oozing from duodenal papilla. Ultrasound revealed right hepatic pseudoaneurysm with large peripheral thrombus and subcapsular haematoma. Gall bladder wall was thickened and lumen was filled with hyperechoic areas consistent with hematoma. Findings were confirmed on the CT angiogram which demonstrates the right hepatic artery aberrant origin from inferior mesenteric artery. Endovascular embolization was successfully using $20 \%$ glue. Bleeding was stopped; blood pressure and heart rate were stabilized. Post-op MRI revealed no evidence of the infarct in the right lobe of the liver. Patient was discharged from hospital after recovery. Follow up after 2 months the patient was asymptomatic.

Posterior circulation pseudoaneurysms are more common in young patients especially after trauma. We have a 17-year-old male patient who presented with severe headache. On plain CT head, there was left medial temporal lobe hematoma with Subarachnoid haemorrhage (SAH). Previous history revealed fall from bicycle 2 months back with no active haemorrhage on CT. Catheter angiogram shows saccular aneurysm in the left Posterior cerebral artery (PCA) P2/P3 junction likely post-traumatic pseudoaneurysm/dissecting aneurysm. Endovascular coiling was performed with occlusion of the aneurysmal sac. Follow up check angiogram at 3 month showed recurrence of pseudoaneurysm which was further managed with parent artery occlusion (Figure 4).

These aneurysms have different etiology than a traditional saccular aneurysm. They are usually seen at the junction of P2/P3 segment which traverses across the tentorium cerebri [6]. Stress on the vessel wall along the edges of tentorium is one possible theory to explain the development of the dissecting aneurysm/pseudoaneurysm. The extra-cranial dissections develop between the media and adventitia layers of vessel wall while the intracranial dissections are mostly present between the intimal and media layers and are surrounded by normal adventitia [7].

Although treatment of dissecting aneurysms/pseudoaneurysms without obliteration of parent artery has been described for aneurysms other than PCA, rarely this approach has been done for dissecting PCA aneurysm
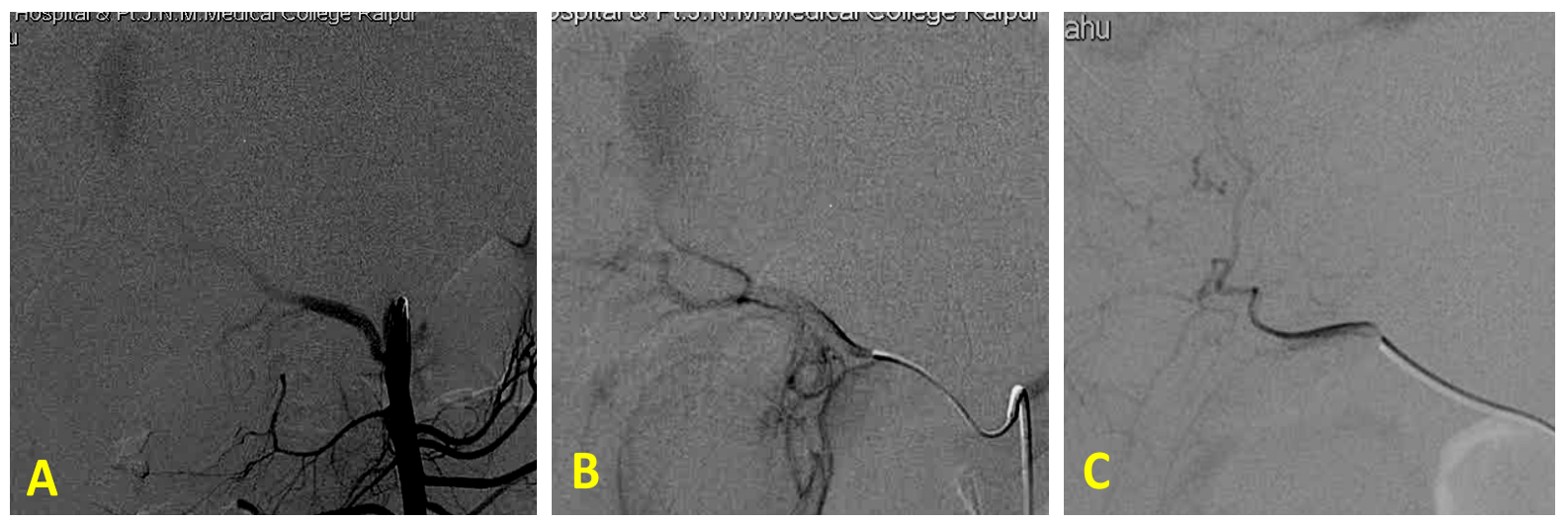

Figure 3. Early arterial and late capillary angiogram (A) and (B) demonstrates large right hepatic artery pseudoaneurysm. Super-selective right hepatic artery angiogram (C) demonstrates complete occlusion of the sac. 

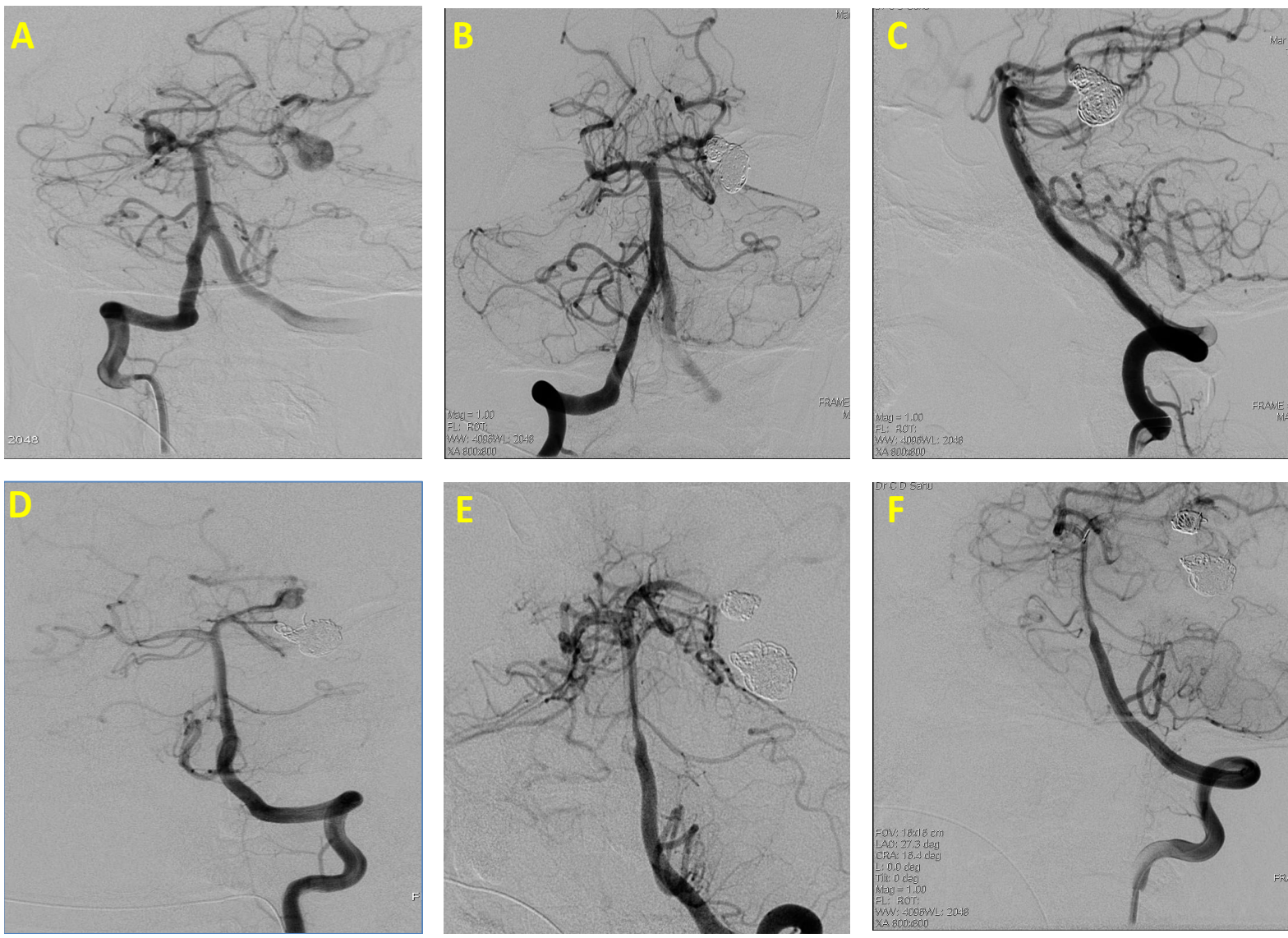

Figure 4. Angiogram (A) shows large left posterior cerebral artery (PCA) pseudoaneurysm. Post endovascular coiling (B) and (C) demonstrate occlusion of the sac. Check angiogram at 3 months shows recurrence of the pseudoaneurysm (D). Later, parent artery occlusion with onyx and coils (E), (F).

(Lempert et al., 1998). We initially attempted this approach in our patients without sacrificing the parent artery. It is likely that the definitive endovascular approach to treat these aneurysms is to occlude the parent artery if the aneurysm is distal to the P2 segment. PCA balloon test occlusion may predict deficits due to artery sacrifice; however, technical difficulty in tracking the balloon must be weighed against the benefit of the test occlusion. Only few of the reported case series are described using a PCA balloon occlusion test [7].

Another 17-year-old male presented at the emergency room with severe headache, prior history reveals trauma 1 week back, initial CT was normal. Repeat CT at ER demonstrates massive SAH. CTA demonstrate supraclinoid ICA dissecting pseudoaneurysm (Figure 5). The location of traumatic intracranial pseudoaneurysms varies, but these are commonly found in the anterior circulation. Reported locations are vertebral artery, internal carotid artery (ICA), anterior cerebral artery (ACA), middle cerebral artery (MCA) and middle meningeal artery. The reported risk of hemorrhage in post-traumatic pseudoaneurysms is $19 \%$, with a peak incidence of rupture at 2 - 3 weeks after the injury, resulting in 32\% - 54\% mortality [8] [9]. Therefore, an early diagnosis and urgent treatment is recommended. Treatment options include surgical repair and endovascular interventions. In our case we performed balloon occlusion test to check the collateral supply followed by endovascular right ICA trapping (Figure 6).

Iatrogenic injuries are one of the common causes of the pseudoaneurysm, we have a 15 -year-old male presented with the history of facial swelling and nasal bleed since 6 months. CT was suggestive of right juvenile nasopharyngeal angiofibroma. Pre-operative embolization was performed with PVA and coil. There was massive post-operative bleeding while removal of the nasal pack. Patient was resuscitated from shock and was taken angiogram suite on urgent basis; angiogram demonstrates right cavernous ICA pseudoaneurysm. However, due to non-availability of the covered stent/flow diverter, endovascular coiling was performed. Small residual neck 


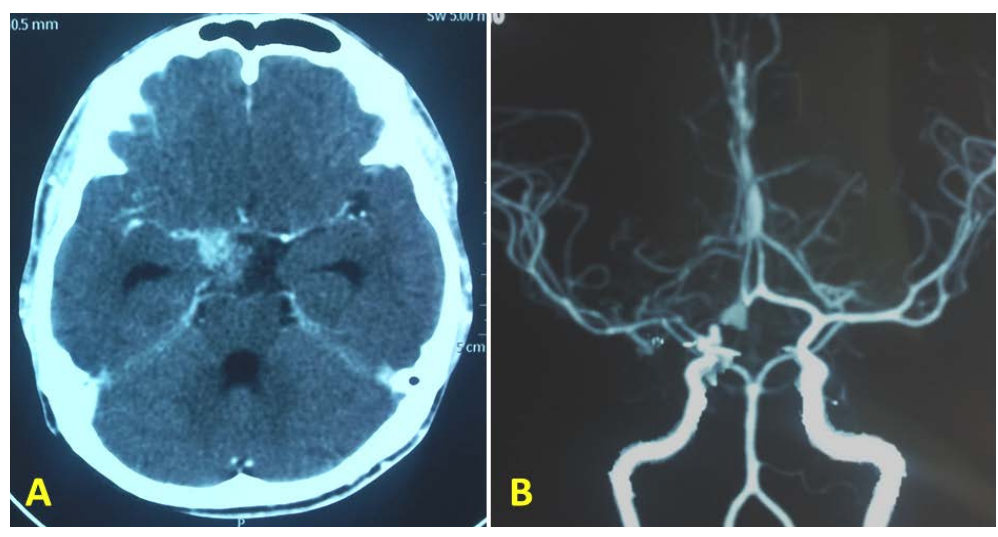

Figure 5. Axial plain CT (A) demonstrates hyperdense enhancing lesion in the right cavernous sinus consistent with pseudoaneurysm. Corresponding CT angiogram (B) shows right supra-clinoid ICA pseudoaneurysm with spasm of the right M1 and M2 MCA branches.
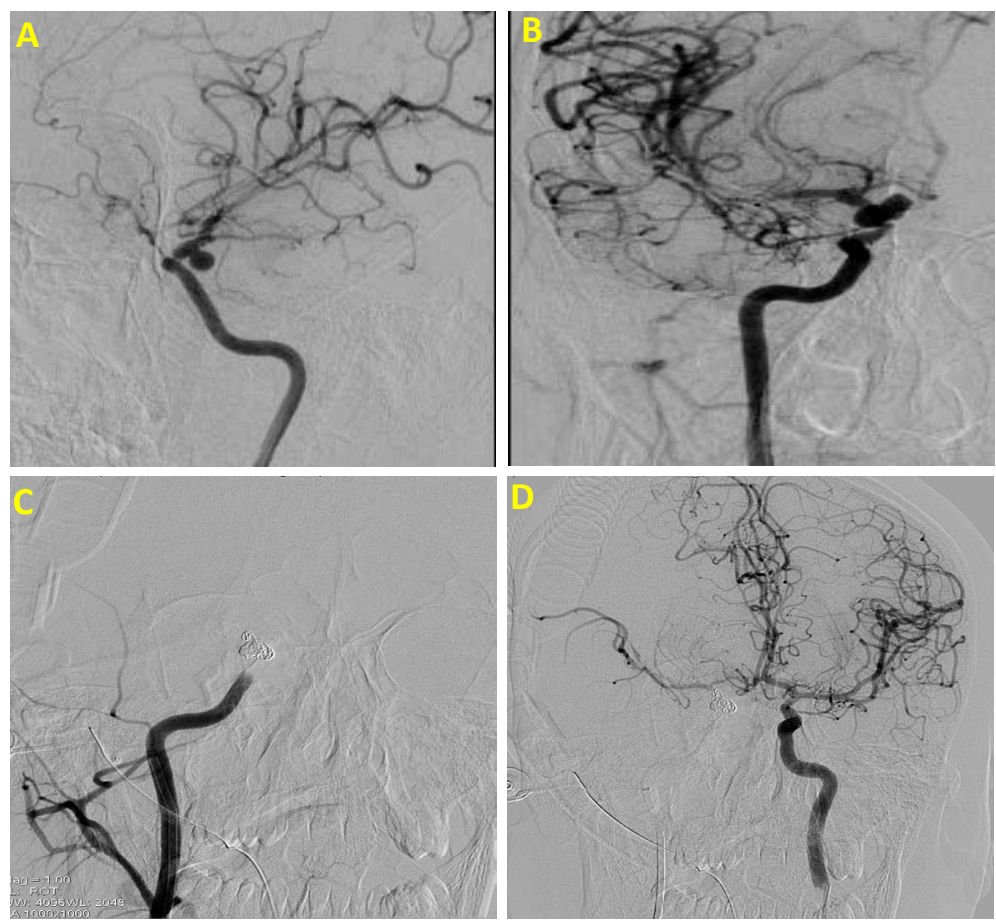

Figure 6. Lateral (A) and AP (B) projection angiograms demonstrates multilobed pseudoaneurysm in the right cavernous ICA. Check angiogram (C) shows endovascular ICA occlusion with coils. Note rigth MCA and ACA (D) filling through the contalateral ICA.

shows refilling, check angiogram at 3 months reveal no significant interval change in the neck refilling (Figure 7).

Frequency of pseudoaneurysms in the upper extremities is much lower than that in the lower extremities. Atherosclerotic aneurysm of the brachial artery is very rare [10]. If the only causal factor is trauma, the aneurysm takes the form of a pseudoaneurysm. Most pseudoaneurysms are the result of penetrating injuries, as it was also in our case (Figure 8).

Treatments option for peripheral artery for pseudoaneurysms that can be performed with ultrasonographicguided manual compression, ligation, endovascular graft implantation, embolization, percutaneous ultrasoundguided thrombin injection (PUGTI), and surgical reconstruction [10]. 

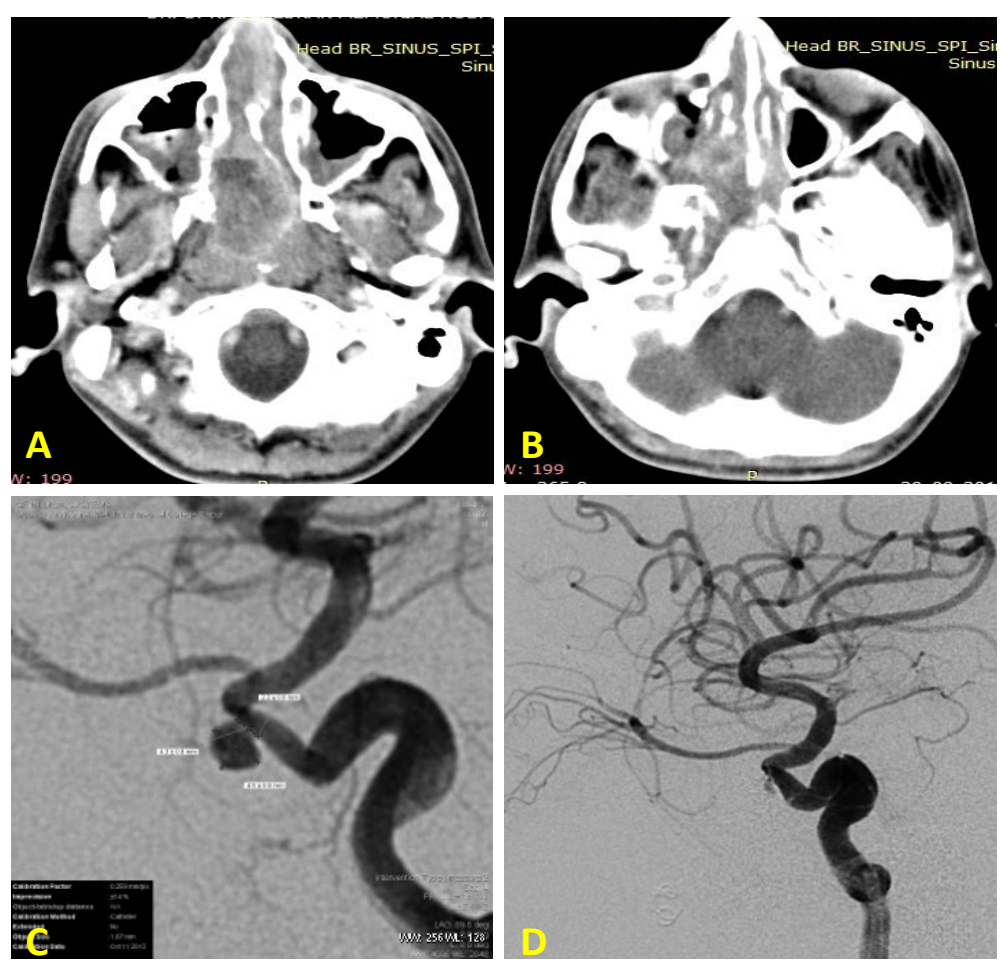

Figure 7. Axial contrast enhanced CT demonstrates large enhancing mass lesion in the naso-pharynx with widening of the pterygo-palatine fissure with anterior displacement of the posterior maxillary antral wall. Angiogram (C) demonstrates small cavernous right ICA pseudoaneurysm. Figure (D) shows occlusion of the pseudoaneurysm with endovascular coiling. Note smalll residual neck.
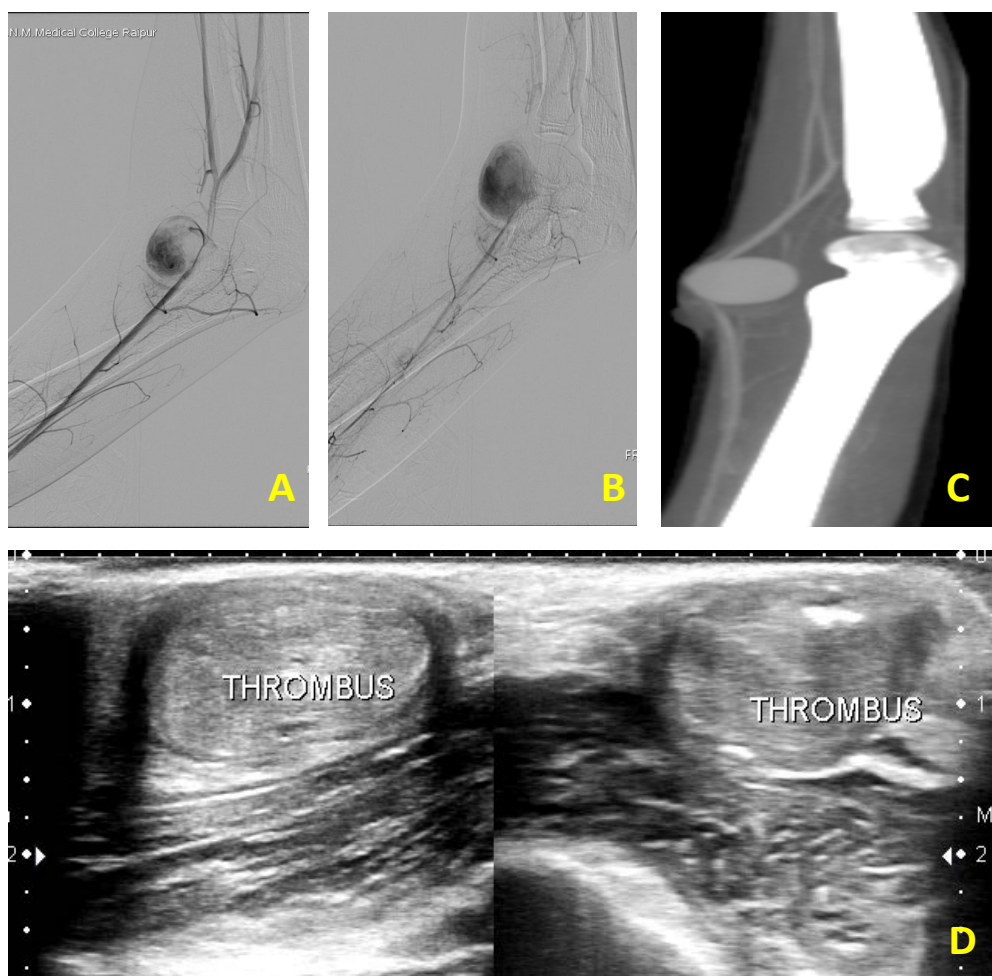

Figure 8. Angiogram (A) and (B) and arterial phase sagittal thin MIP CT (C) demonstrates large brachial artery pseudoaneurysm. Post embolization Ultrasound (D) show hyperechoic area within the sac consistent with thrombus. 
Nowadays PUGTI, technically less invasive, was introduced for treating pseudoaneurysm of the injured peripheral artery. Thrombin, a serine protease, cleaves fibrinogen into fibrin monomers, enables the formation of cross-linked fibrin by activating Factor XIII, and stimulates platelet activation [10]. When thrombin is exposed to a relatively static pool of blood in a pseudoaneurysm, it produces rapid thrombosis. Reeder et al employed low-dose (100 U/cc) thrombin injection to treat iatrogenic femoral artery pseudoaneurysm [10].

The special characteristic of brachial artery pseudoaneurysm in our case is its relatively wide aneurysmal neck that is prone to intra-arterial thrombosis or distal embolization of new thrombus during PUGTI. To prevent these dreadful complications, the volume of the injected thrombin into the aneurysmal sac must not be higher than that of active blood flow in the pseudoaneurysm. In addition, the needle tip must be guided by CDUS to keep it away from the aneurysmal neck.

Major complications of PUGTI are anaphylaxis, abnormal haemostasis, intra-arterial thrombosis, and distal embolization. Intra-arterial thrombosis and distal embolization were treated by 5000 unit heparin infusion and keeping the forearm warm using warm sponging. Intra-arterial thrombosis and distal embolization were treated by 5000 unit heparin infusion and keeping the forearm warm using warm sponging.

Another 23-year-old female patient, presented with left forearm pulsatile swelling no definite history of trauma. CDUS demonstrate left ulnar artery pseudoaneurysm. Manual graded ultrasonographic compression was given for occlusion of the pseudoaneurysm sac [11].

Percutaneous renal procedures could lead to several reno-vascular injuries such as hematomas, arterio-venous fistulas or pseudoaneurysm. The reported incidence of renal pseudoaneurysm following percutaneous nephrolithotomy (PCNL) is $0.6 \%$ to $1 \%$ [3] and it is usually assessed by renal angiography. Selective renal embolization is currently considered the most appropriate technique for the treatment for these complications [3]. We report two cases of renal pseudoaneurysm following PCNL, successfully treated by endovascular-occlusion using n-BCA and microcoils (Figure 9).

Selective renal embolization is currently considered as the most appropriate technique in the treatment for reno-vascular complications with a success rate greater than $80 \%$ and low complication rate [3].

The splenic artery is the most common site for pseudoaneurysms secondary to pancreatitis. Treatment of splenic artery aneurysm is recommended in patients with aneurysms greater than $2 \mathrm{~cm}$ [12]. The prevalence of splenic artery aneurysm has increased in patients with portal hypertension and is estimated at $7 \%-20 \%$ with trauma \& pancreatitis [13]. Here we report a similar case in which the patient presented with splenic artery pseudoaneurysm which was ruptured, \& presented with hematemesis \& malena (Figure 10). Surgical mortality is approximately $16 \%$ for pseudoaneurysms in the head of the pancreas and $50 \%$ for lesions in the tail. The risk of spontaneous rupture of extra-organic visceral pseudo aneurysm is very high regardless of their size and the mortality rate for such ruptures in morbid postsurgical patients approaches to about 100\% [14].

Selective embolization of the pseudoaneurysm is preferred although splenic artery endovascular occlusion is an alternative option, if selective catheterization of the aneurysm cannot be performed. In our case, we deployed
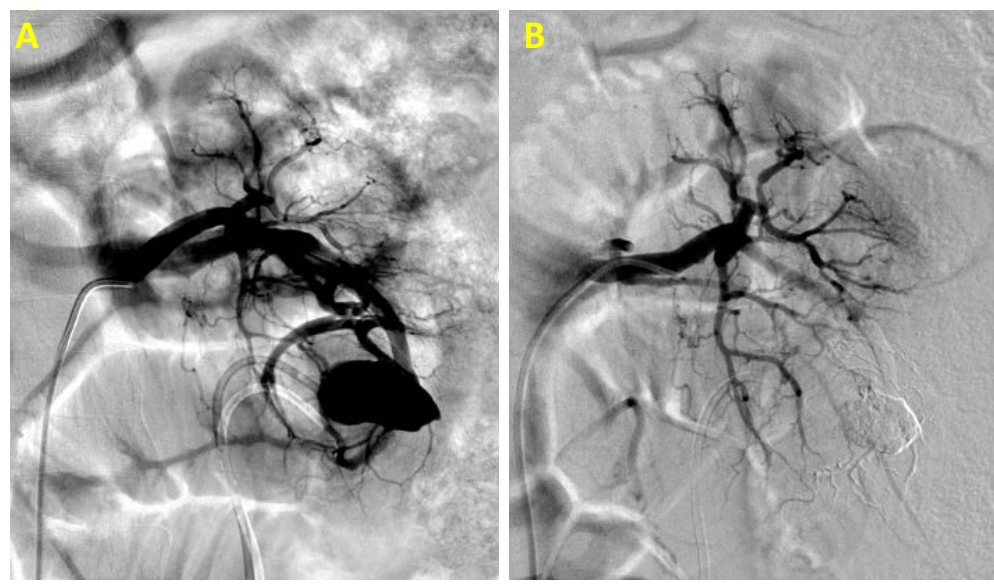

Figure 9. Renal angiogram shows large pseudoaneurysm in a branch of left renal artery. (B) Occlusion of the pseudoaneurysm and its proximal feeding artery. 

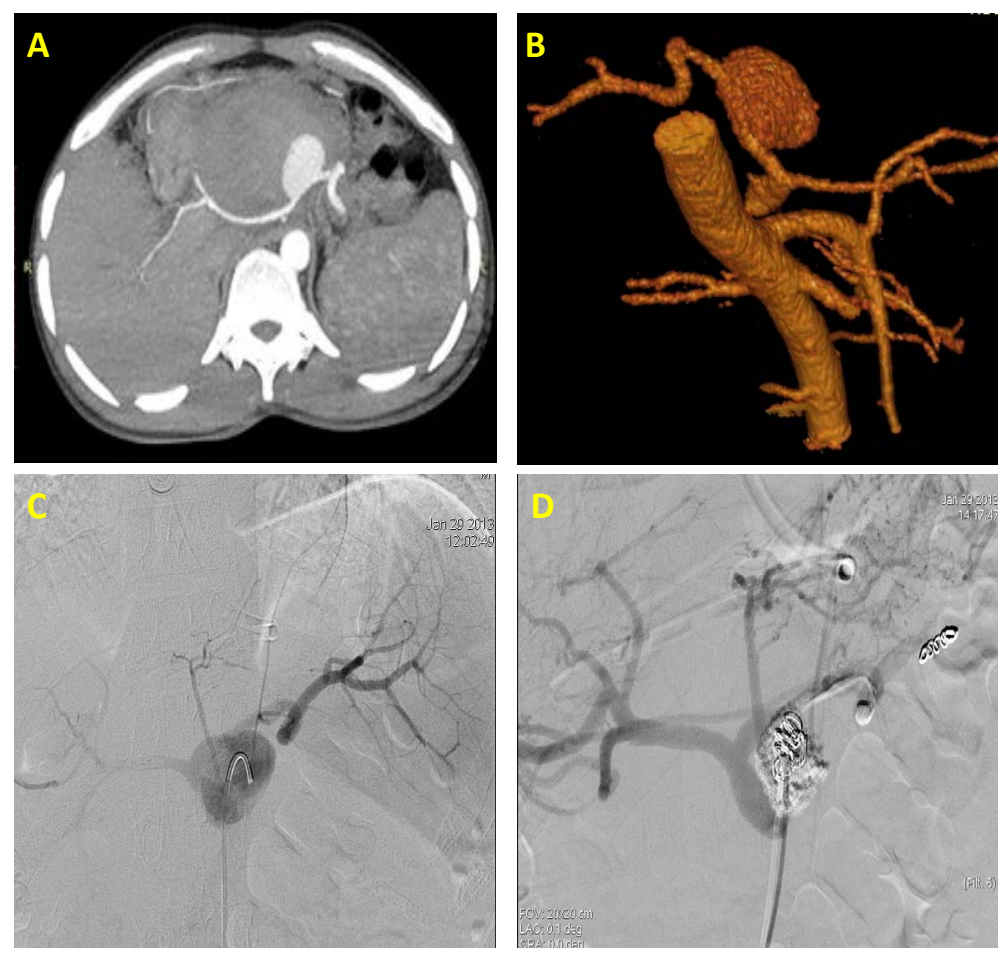

Figure 10. Arterial phase axial thin CT-MIP (A) and VRT (B) CTA showing large splenic artery pseudoaneurysm. Angiogram (C) demonstrate splenic artery pseudoaneurysm. Check angiogram (D) demonstrates complete occlusion of the pseudoaneurysm with glue and coils, also note distal migration of the coils and glue in the splenic artery.

initial few micro-coils followed by glue in the pseudoaneurysmal sac to achieve occlusion. However, there was some spillage of glue into the distal most splenic artery \& its branches. Follow-up CT of the patient demonstrated small clinically silent peripheral splenic infarcts.

The occurrence of complications after endovascular treatment is uncommon. Possible complications include post-embolization syndrome, transient elevation of pancreatic enzymes, splenic infarction, infection, abscess, and, rarely rupture of a pseudoaneurysm. Follow up imaging with CT or CDUS is recommended for assessment of the adequacy of treatment.

With increasing incidence of pancreatitis induced pseudoaneurysm, one of the most common presentation is haemosuccus pacreaticus due to rupture of aneurysm/pseudoaneurysm of peri-pancreatic artery into pancreatic duct [15]. The incidence of pseudoaneurysms in patients with severe acute pancreatitis is up to $10 \%$ [16]. Gastroduodenal artery aneurysms are extremely rare, comprising only $1.5 \%$ of all reported visceral aneurysms [16]. The CT demonstration of a homogeneously enhancing structure within or adjacent to a pseudocyst or contiguous with a vascular structure in a case of pancreatitis is highly suggestive of an associated pseudoaneurysm.

The visceral artery aneurysm recanalization rate following embolization is $9 \%$ [16]. It is thus important to follow-up such patients on short term basis, as recanalization rates are high which happened in our gastroduodenal pseudoaneurysm case (Figure 11). In this case first we injected percutaneous human thrombin. The pseudoaneurysm sac recanalized after three days, following which we performed endoluminal trapping of the sac.

We report a patient with a pseudoaneurysm of a transverse facial artery following history of assault by stick over the left side of the face, developed a massive hematoma over the face. The following day, he was admitted to emergency room at our hospital. Computed tomography revealed a large subcutaneous hematoma. Selective External Carotid Artery (ECA) angiogram shows small pseudoaneurysm in the left transverse facial artery, trans-arterial embolization was performed with $20 \%$ glue (Figure 12) [17].

Another patient presented with hypovolemic shock in the emergency department following caesarean section. Urgent laprotomy demonstrates massive retroperitoneal and pelvic haematoma with necrosis of right fallopian 

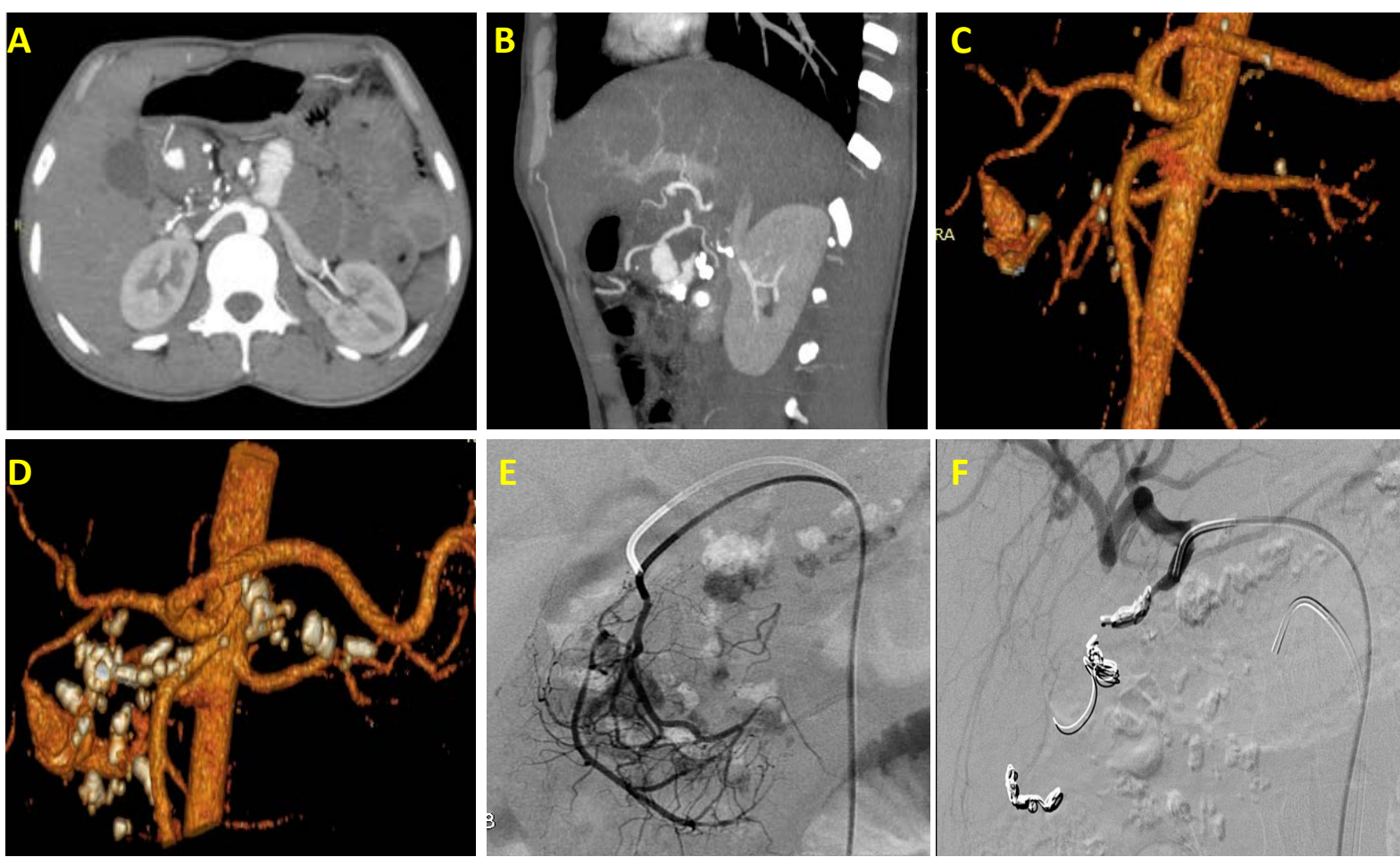

Figure 11. Arterial phase thin CTA-Maximum Intensity Projection (MIP) axial (A), sagittal (B), oblique Volume Rendering Technique (VRT) (C) \& (D) demonstrates gastroduodenal artery pseudoaneurysm with eccentric thrombus, multiple punctuate calcifications in the pancreas representing changes associated with chronic pancreatitis. Digital angiogram shows gastroduodenal pseudoaneurysm. Post-embolization check angiogram demonstrates occlusion of the proximal and distal gastroduodenal artery with no reconstitution of the pseudoaneurysm.
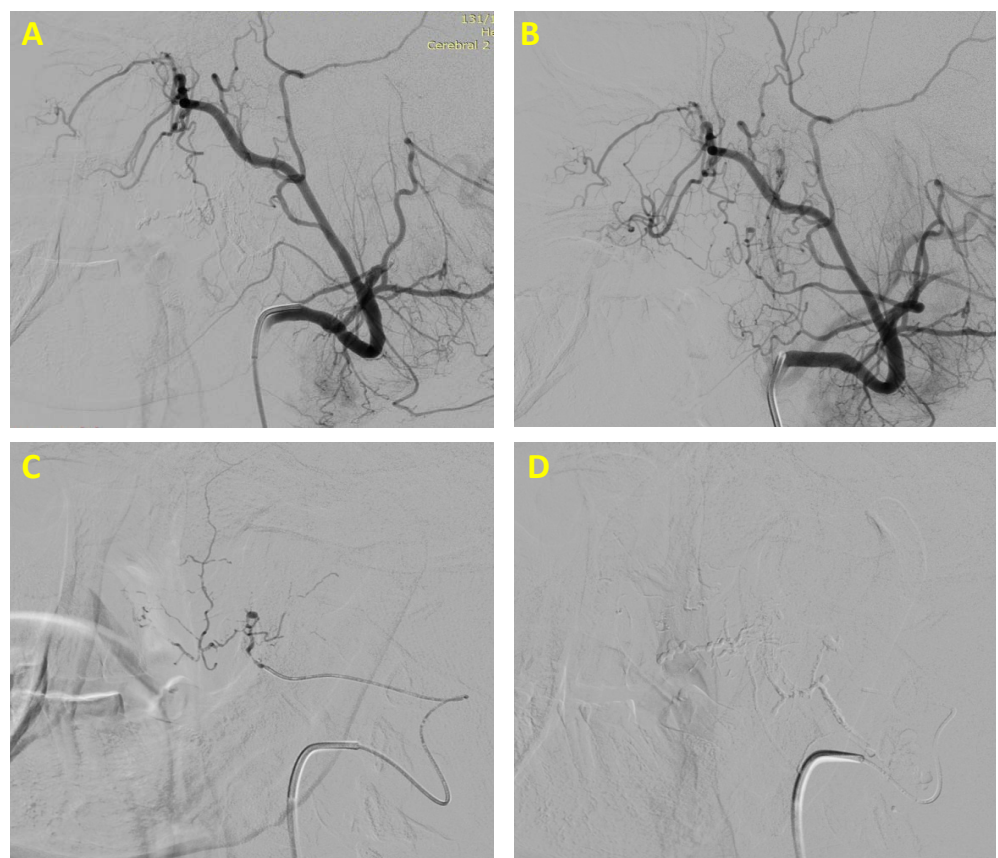

Figure 12. Left ECA angiogram (A) \& (B) demonstrates small pseudoaneurysm in the branch of the facial artery. Super-selective injection (C) shows small pseudoaneurysm indicated by arrow. Check angiogram (D) shows complete occlusion of the facial artery. 
tube; there was no definite arterial leak which prompts us for an urgent diagnostic angiogram. Angiogram demonstrates pseudoaneurysm in a right internal iliac artery branch pseudoaneurysm, which was successfully embolized with 20\% glue (Figure 13) [18].

Iatrogenic pseudoaneurysms are very common in the modern era. One of our patients presented with postappendicectomy fourth day massive malena which raise the suspicion of the vascular injury. Patient was immediately taken for catheter angiogram, which reveals large pseudoaneurysm in the ilio-colic branch of the superior mesenteric artery. Emergent endovascular trapping was performed with microcoils. Following procedure patient was stable with no fresh malena (Figure 14).

\section{Conclusion}

Complications of pseudoaneurysm need early diagnosis and prompt treatment. It needs high index of suspicion on CDUS and CECT. Catheter angiogram was done for further catheterization and endovascular management.
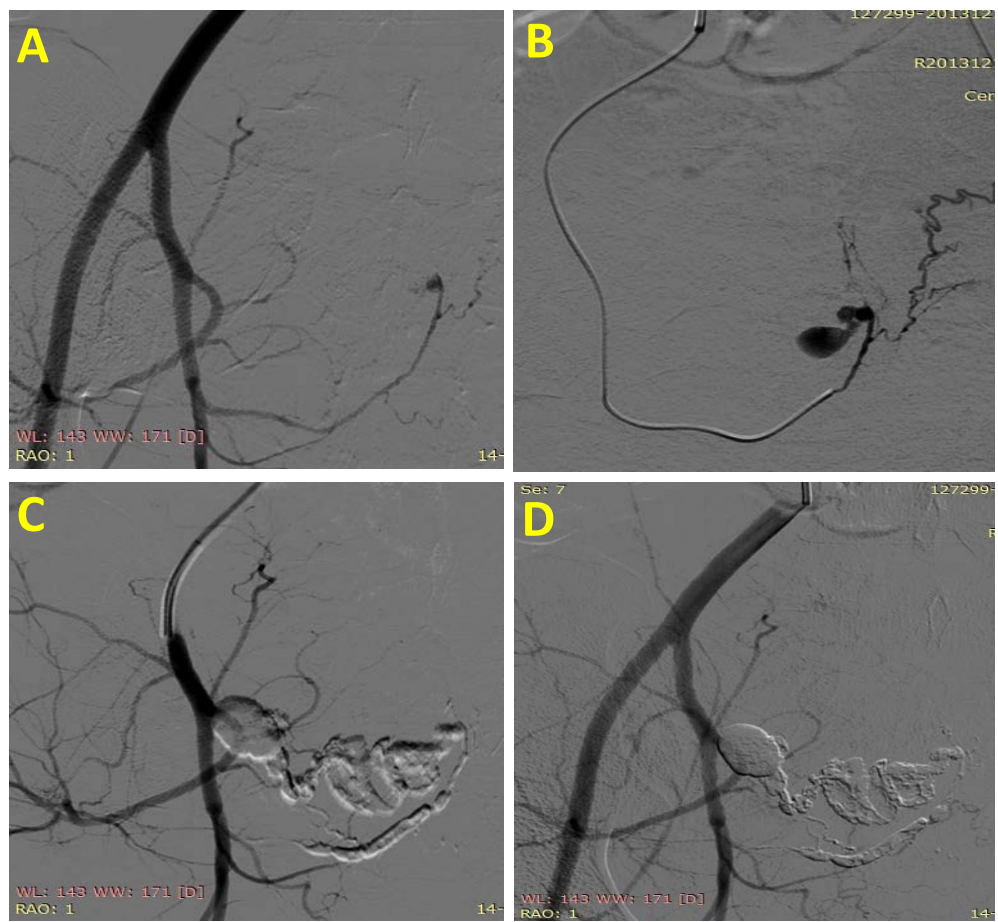

Figure 13. Angiogram (A) \& (B) demonstrates in a rigth internal iliac artery pseudoaneurysm. Check angiogram (C) \& (D) demonstrates occlusion of the feeding artery.
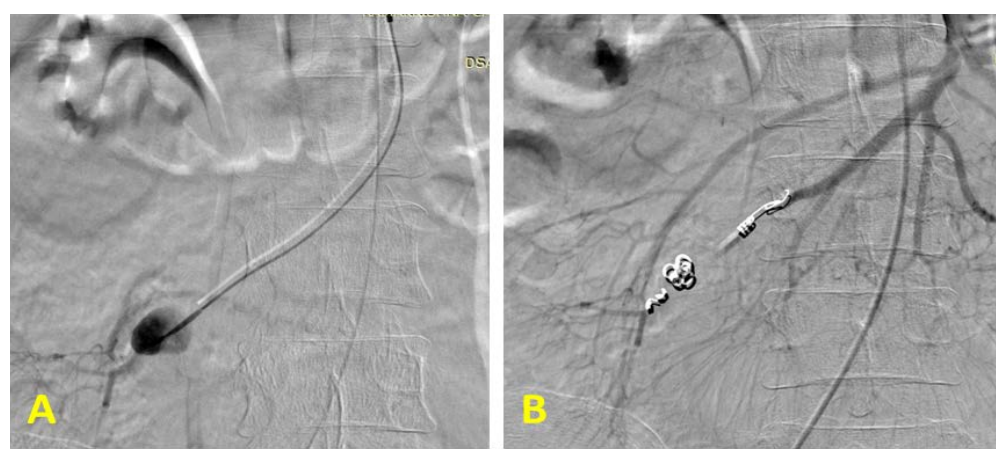

Figure 14. Angiogram (A) demonstrates pseudoaneurysm of ilio-colic artery and (B) shows endovascular trapping of the pseudoaneurysm with coils, no distal reconstitution. 
These procedures are minimally invasive technique to exclude the pseudoaneurysm from the circulation. It reduces the possibility of life threatening internal hemorrhage, and nowadays is a preferred procedure over surgery.

\section{References}

[1] http://en.wikipedia.org/wiki/Pseudoaneurysm

[2] Saad, N.E.A., Saad, W.E.A., Davies, M.G., David, L., Fultz, W.P.J. and Rubens, D.J. (2005) Pseudoaneurysms and the Role of Minimally Invasive Techniques in Their Management. RadioGraphics, 25, S173-S189. http://dx.doi.org/10.1148/rg.25si055503

[3] Soyer, P., Desgrippes, A., Vallee, J.N. and Rymer, R. (2000) Intrarenal Pseudoaneurysm after Percutaneous Nephrostolithotomy: Endovascular Treatment with N Butyl-2-Cyanoacrylate. European Radiology, 10, 1358. http://dx.doi.org/10.1007/s003300000317

[4] Sansonna, F., Boati, S., Sguinzi, R., Migliorisi, C., Pugliese, F. and Pugliese, R. (2011) Severe Hemobilia from Hepatic Artery Pseudoaneurysm. Case Reports in Gastrointestinal Medicine, 2011, Article ID: 925142.

[5] Singh, C.S., Giri, K., Gupta, R., Aladdin, Md. and Sawhney, H. (2006) Successful Management of Hepatic Artery Pseudoaneurysm Complicating Chronic Pancreatitis by Stenting. World Journal of Gastroenterology, 12, 5733-5734.

[6] Lazinski, D., Willinsky, R.A., TerBrugge, K. and Montanera, W. (2000) Dissecting Aneurysms of the Posterior Cerebral Artery: Angioarchitecture and a Review of the Literature. Interventional Neuroradiology, 42, 128-133. http://dx.doi.org/10.1007/s002340050031

[7] Taqi, Md., Lazzaro, M.A., Pandya, D.J., Badruddin, A. and Zaidat, O. (2011) Dissecting Aneurysms of Posterior Cerebral Artery: Clinical Presentation, Angiographic Findings, Treatment, and Outcome. Frontiers in Neurology Endovascular and Interventional Neurology, 2, 38.

[8] Miley, J.T., Rodriguez, G.J. and Qureshi, A.I. (2008) Traumatic Intracranial Aneurysm Formation Following Closed Head Injury. Journal of Vascular and Interventional Neurology, 1, 79-82.

[9] Matouk, C.C., Kaderali, Z., terBugge, K.G. and Willinsky, R.A. (2012) Willinsky Long-Term Clinical and Imaging Follow-Up of Complex Intracranial Aneurysms Treated by Endovascular Parent Vessel Occlusion. American Journal of Neuroradiology, 33, 1991-1997. http://dx.doi.org/10.3174/ajnr.A3079

[10] Yetkin, U. and Gurbuz, A. (2003) Post-Traumatic Pseudoaneurysm of the Brachial Artery and Its Surgical Treatment. Texas Heart Institute Journal, 30, 293-297.

[11] Hajarizadeh, H., LaRosa, C.R., Cardullo, P., Rohrer, M.J. and Cutler, B.S. (1995) Worcester Mass Ultrasound-Guided Compression of Iatrogenic Femoral Pseudoaneurysm Failure, Recurrence, and Long-Term Results. Journal of Vascular Surgery, 22, 425-433. http://dx.doi.org/10.1016/S0741-5214(95)70010-2

[12] Agrawal, G.A., Johnson, P.T. and Fishman, E. (2007) Splenic Artery Aneurysms and Pseudoaneurysms: Clinical Distinctions and CT Appearances. AJR, 188, 992-999. http://dx.doi.org/10.2214/AJR.06.0794

[13] Madoff, D.C., Denys, A., Wallace, M.J., Murthy, R., Gupta, S., Pillsbury, E.P., Ahrar, B.A.K., Bessoud, B. and Hicks, M.E. (2005) Splenic Arterial Interventions: Anatomy, Indications, Technical Considerations, and Potential Complications. RadioGraphics, 25, S191-S211. http://dx.doi.org/10.1148/rg.25si055504

[14] Taori, K., Rathod, J., Disawal, A., Mundhada, M., Rewatkar, A., Bakare, V., Wavare, P. and Puria, R. (2013) Endovascular Embolization of Pseudoaneurysms Complicating Pancreatitis Using Microcoils: Case Series. Open Journal of Radiology, 3, 33-40. http://dx.doi.org/10.4236/ojrad.2013.31005

[15] Koren, M., Kinova, S., Bedeova, J., Javorka, V., Kovacova, E. and Kekenak, L. (2008) Hemosuccus Pancreatitis. Bratislavské Lekárske Listy, 109, 37-41.

[16] Chong, W.W., Tan, S.G. and Htoo, M.M. (2008) Endovascular Treatment of Gastroduodenal Artery Aneurysm. Asian Cardiovascular \& Thoracic Annals, 16, 68-72. http://dx.doi.org/10.1177/021849230801600118

[17] Germiller, J.A., Myers, L.L., Harris, M.O. and Bradford, C.R. (2001) Pseudoaneurysm of the Proximal Facial Artery Presenting as Oropharyngeal Hemorrhage. Head \& Neck, 23, 259-263. http://dx.doi.org/10.1002/1097-0347(200103)23:3<259::AID-HED1027>3.0.CO;2-4

[18] Nanjundan, P., Rohilla, M., Raveendran, A., Jain, V. and Khandelwal, N. (2011) Pseudoaneurysm of Uterine Artery: A Rare Cause of Secondary Postpartum Hemorrhage, Managed with Uterine Artery Embolisation. Journal of Clinical Imaging Science, 1, 14. 
Scientific Research Publishing (SCIRP) is one of the largest Open Access journal publishers. It is currently publishing more than 200 open access, online, peer-reviewed journals covering a wide range of academic disciplines. SCIRP serves the worldwide academic communities and contributes to the progress and application of science with its publication.

Other selected journals from SCIRP are listed as below. Submit your manuscript to us via either submit@scirp.org or Online Submission Portal.
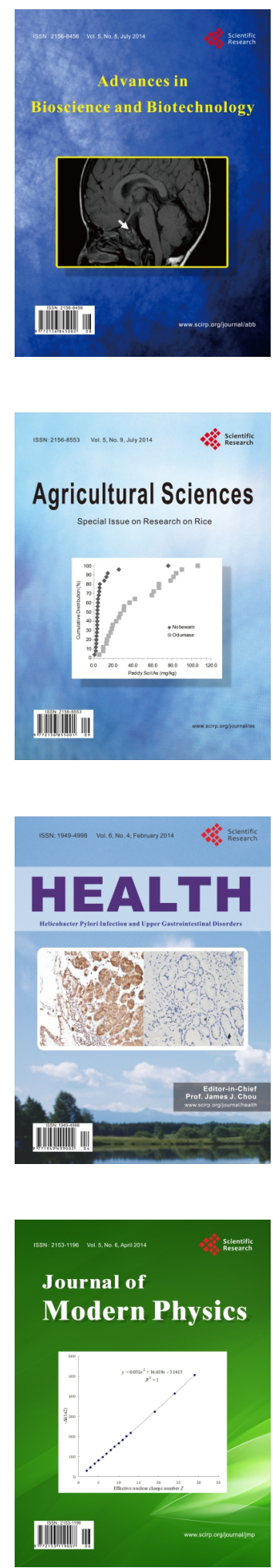
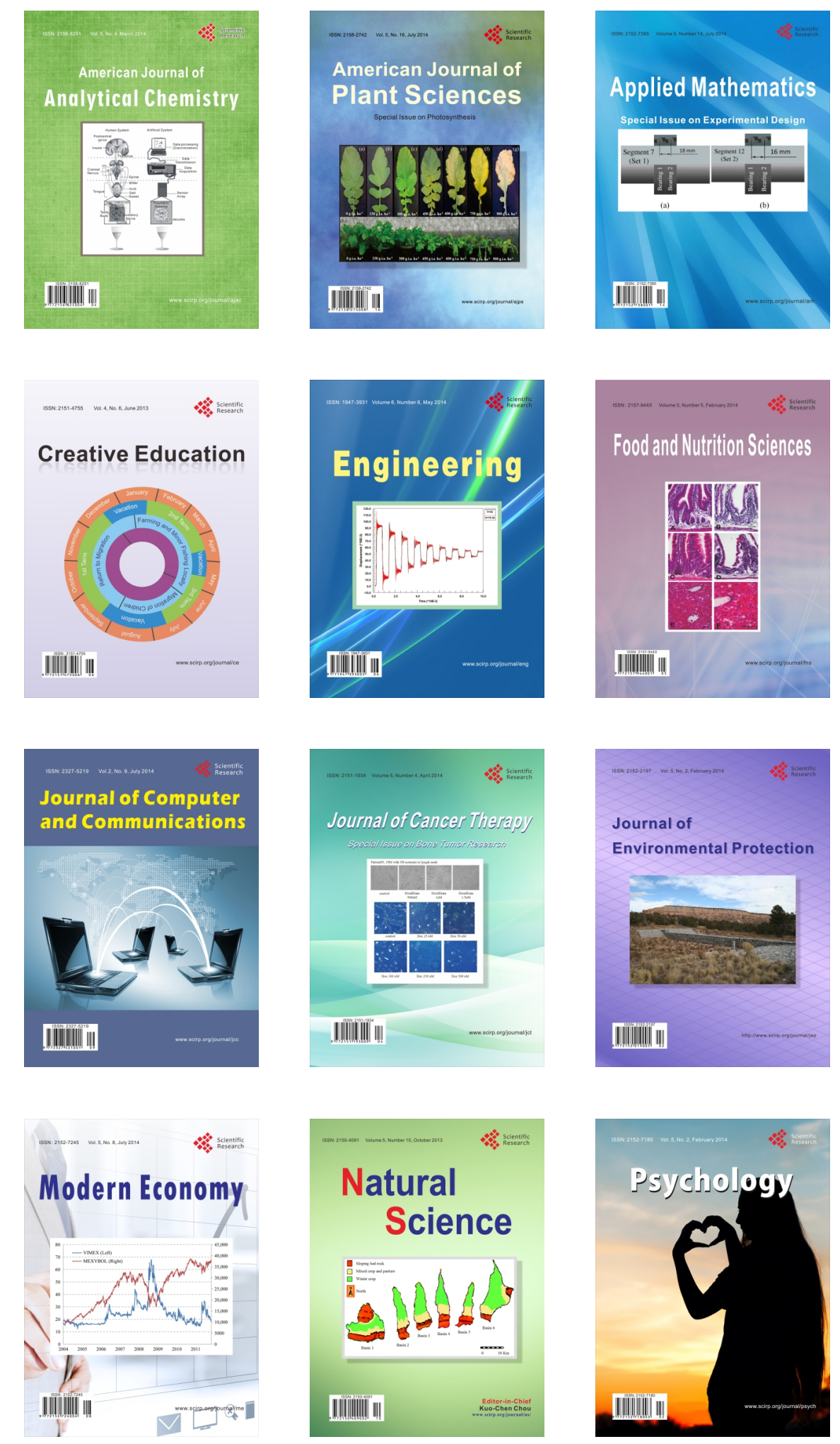